most varied interests, retired, and Dr. J. F. Payne, of St. Thomas's Hospital, joined as co-editor, thus maintaining the link with medical microscopy. With Vol. 16, 1876, E. Ray Lankester became chief editor, but had a varying panel of collaborators chosen from the leading biologists of the country. There followed a period of remarkable growth, which reached a peak in 1885, during which year $912 \mathrm{pp}$. of text and 62 plates appeared, and a second peak in 1894, with 904 pp. and 84 plates. The former was towards the end of a period when the microscope had greatly facilitated the study of the invertebrates. By this time the Journal was without equal in Great Britain and had achieved a world-wide reputation. Behind much of this work was the background of the idea of evolution, and there grew up a sort of unity, lacking in the early years, in which each piece of morphology or embryology seemed to have a place.

The second peak was in part associated with the extended use of the microtome and the rise of vertebrate embryology, again with a distinctly evolutionary background, which occupied an increasing amount of space but not to the exclusion of the invertebrates, particularly Protozoa. During this expansion period the Journal of Botany and other specialist journals appeared, so that the "Q.J." became more and more zoological. This tendency to restrict the scope of $a_{b}$ publication seems to be inevitable; but it is to be hoped that it will not be carried to the extent that the Quarterly Journal becomes another of those journals not looked at save by workers in its own narrow field. E. Ray Lankester: remained editor until Vol. 64, 1919, by which time he had contributed seventy-eight articles to it, a number of them becoming classics. He retired from active participation; but his name appeared as honorary editor until his death in 1929. Vol. 64 also marked fifty years of his editorship, and this was commemorated by Prof. G. C. Bourne in a long review of the outstanding memoirs that had appeared and the many distinguished names, both British and foreign, that had been associated with the Journal. He directed attention to one of the principal features of its outstanding success, the universality of its appeal, and that while it "had its specialities it has never been specialised". The new editor was Prof. E. S. Goodrich, a pupil of Lankester's at University College, London, whom he took with him when he went to the Linacre chair at Oxford. Goodrich's first contribution to the Journal was in 1893, and like his predecessor he was to prove a great editor and a great master of zoology. Three parts of Vol. 86, 1946, are taken up by a review of the work, largely by himself and his pupils, on the cœlom, genital ducts and nephridia that had appeared in the fifty years since his initial contribution to the problem. He died shortly after he had corrected the proofs of this, and before it had appeared in print, and so ended a connexion of fifty-two years with the Journal, for the last twenty-five of which he had been editor.

With the death of Prof. Goodrich the ownership of the Journal changed hands, its publication was taken over by the Clarendon Press, and Dr. C. F. A. Pantin, who had joined the panel of collaborators in 1939, and Dr. John Baker became joint editors. A comparison between the earliest and the latest volumes is interesting, and shows that a number of similar problems are investigated. The techniques, of course, differ, for the plain microscope is replaced by the electron and phase-contrast microscopes, etc., freeze drying and refined chemical (including staining) methods. It is essential to explore the possibilities and limitations of new tech. niques; but it seems to follow that the various contributions tend to lack the coherence so noticeable in the years before and after the turn of the century. It is interesting, too, in view of the present extensive use of photomicrographs, to find that this method of illustration was used for the first time, so far as has been traced, in one of the plates in the first volume of the Quarterly Journal of Microscopical Science.

The Journal is to be congratulated on its century and on the fact that it is "still going strong" ; may it long continue to be the fertile source of inspiration that it has been in the past.

\section{INORGANIC CHEMICAL KINETICS AND REACTIONS}

$\triangle$ CHEMICAL SOCIETY symposium generally A takes the form of a sort of scientific stocktaking at which progress and achievement in some chosen field are reported and the contemporary position impartially assessed. The symposium on "Inorganic Kinetics and Reactions", held at Burlington House, London, on February 4, was arranged by Dr. J. Chatt and Dr. L. E. Sutton rather to direct attention to a field of rapidly growing importance, and in his introductory remarks the president of the Society, Prof. C. K. Ingold, urged his many listeners to avail themselves of the opportunities open for British chemists to take their full share and-as he hoped--a leading part in its development.

The first paper, by Dr. H. M. N. H. Irving (Oxford), took the form of a general survey of problems and methods. After paying a tribute to Prof. Ingold for the great part he had played in raising our knowledge of the kinetics of organic reactions to such an advanced state, Dr. Irving suggested that the comparatively undeveloped state of inorganic kinetics is not due entirely to the general neglect-particularly in Britain - of inorganic chemistry as a whole, nor to the fact that inorganic reactions are often fast, for techniques for dealing with this problem are available. But while the organic chemist generally has a precise knowledge of the concentrations, compositions and detailed structures of the materials reacting in the systems he investigates, remarkably little is known in many cases concerning even the actual species which participate in an inorganic reaction such as that formally represented by the equation :

$$
\mathrm{Sn}^{2+}+2 \mathrm{Fe}^{3+} \rightleftharpoons \mathrm{Sn}^{4+}+2 \mathrm{Fe}^{2+} \text {. }
$$

The extent to which a cation is hydrated in solution, the firmness with which the primary solvation sheath is attached, the extent and rate of ionization of hydrogen ions from an aquo-cation to form watersoluble basic species or polynuclear complexes, and the effects of complex formation with all anions present-these factors are seldom known with any accuracy, though they may be of essential significance in elucidating reaction mechanisms and kinetics in inorganic chemistry.

Techniques for measuring the rates of inorganic reactions are already numerous, though dynamic flow methods and calorimetric methods have not 
yet been extensively employed. Bjerrum's effective device of working at very low temperatures $\left(-75^{\circ}\right.$ to $-100^{\circ} \mathrm{C}$.) has already provided interesting results. Great impetus has recently been given to the field by the general availability of radioactive and stable isotopes and ancillary counting equipment and mass-spectrographs.

A feature common throughout inorganic kinetics is the complexity of the processes taking place. For the exchange reaction of ferrous and ferric ions the rate equation may be written :

$$
R=\left[\mathrm{Fe}^{\mathrm{II}}\right]\left[\mathrm{Fe}^{\mathrm{III}}\right]\left\{k_{1}+k_{2} /\left[\mathrm{H}^{+}\right]+k_{X}\left[\mathrm{X}^{-}\right]\right\},
$$

where the inverse rate-dependence on hydrogen-ion concentration shows that the (hydrated) species $\mathrm{FeOH}++$ is involved, while the term involving $X^{-}\left(=\mathrm{Cl}^{-}, \mathrm{NO}_{3}^{-}\right.$, etc. $)$shows that ferric complexes of the type $\mathrm{FeX}^{++}$are participating. But while the kinetics can be interpreted by postulating a transition state of the type (III) $\mathrm{Fe}^{++}$$\mathrm{OH}$... $\mathrm{Fe}(\mathrm{II})^{++}$, the effective exchange of charge which appears to take place may involve (i) actual transfer of an electron from right to left, (ii) transfer of a hydroxyl group, or (iii) transfer of a proton from a water molecule attached to the ferrous ion to the hydroxyl group.

Exchange of a complex $M I_{n}$ with a labelled ligand $L^{*}$ may proceed either through dissociation (with or without an intermediate stage of aquation) or by an association process which involves an increase in the co-ordination number of the central atom. Examples of both types are known, and considerable progress has been made in correlating the ease of exchange with the type and strength of bonding in the complex. In organic chemistry, stereochemistry is dominated by the tetravalency of the carbon atom ; but in inorganic chemistry, planar, tetrahedral and octahedral dispositions provide a variety of new problems, some of which would be dealt with in the next paper. As an illustration of the unsolved problems of inorganic mechanism, Dr. Irving concluded by quoting the reaction of $D-\left[\mathrm{CoCO}_{3}(l \cdot p n)_{2}\right]^{+}$with a 100 per cent excess of $d$-l-tartaric acid whereby the dextro-form displaces carbonate ion preferentially from the complex, thus effecting a partial resolution. Is this due to differing stabilities of the complexes containing $d$ - or $l$-tartaric acid, or to different rates of reaction of the two enantiomorphs with the carbonatocobaltic ion ?

Prof. C. K. Ingold (London) then spoke on the "Mechanism and Stereochemistry of Octahedral Substitution", dealing first with the kinetics of chlorine displacement from $l$-cis-dichlorobisethylenediaminecobalt-III by a graded series of nucleophilic reagents $\left(X^{-}\right)$such as $\mathrm{MeO}^{-}, \mathrm{N}_{3}^{-}, \mathrm{NO}_{2}^{-}$, SCN-, $\mathrm{Br}^{-}, \mathrm{Cl}^{-}$and $\mathrm{NO}_{3}^{-}$. Kinetics were followed by variety of techniques such as polarimetry, spectrophotometry, titrimetry, and the use of radioactive chlorine-36. While bimolecular reactions occur with the first three reagents named, nucleophilic substitution proceeds by $S_{N} l$ mechanism with the last four. Since optical activity is completely lost, and at the same rate, in these last four cases, the following mechanism was proposed by Prof. Ingold :

$\left[\mathrm{CoEn}_{2} \mathrm{Cl}_{2}\right]_{\text {S.low, rate controlling }}^{+} \underset{2}{\stackrel{-\mathrm{Cl}^{-}}{\longrightarrow}}\left[\mathrm{CoEn}_{2} \mathrm{Cl}\right]^{+}+\underset{\text { rapid }}{\stackrel{X^{-}}{\rightarrow}}\left[\mathrm{CoEn}_{2} \mathrm{ClX}\right]^{+}$
The short-lived, optically inactive intermediate contains 5-covalent cobalt-III and may be formulated as a triangular bipyramid or as a square pyramid. It can take up the substituting agent at comparably rapid rates in all the distinguishable five positions in proportions which depend in detail on the groups already present, and on the nature of that entering.

Prof. Ingold pointed out thet a most confusing variety of stereochemical changes among co-ordination compounds of the type $\operatorname{Co} R_{1} A X$ has been reported on replacing $X$ by $Y$. If these are bimolecular and proceed by edge-displacement, the type of configurational change observed depends essentially upon the atom chosen as the reference point. If this were atom 1 , the edge-displacement could be described as a cis $\rightarrow$ trans change; if at 2 , as trans $\rightarrow$ cis; if at 3 or 4 , as either
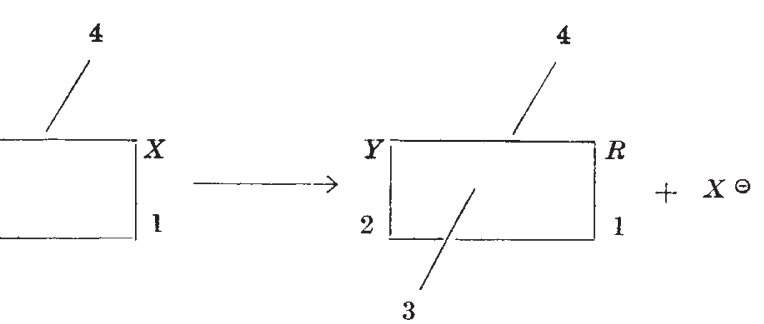

$d \rightarrow l$, or $l \rightarrow d$. Indeed, if certain geometrical conditions are satisfied, edge-displacement can proceed without apparent geometrical isomerization or change of optical configuration. In two cases the steric course of an $S_{N} 2$ change has been studied in detail, with the following results :

$$
\begin{aligned}
& \text { per cent per cent }
\end{aligned}
$$

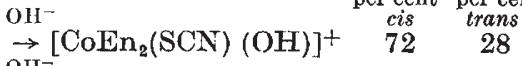

$$
\begin{aligned}
& +\stackrel{\mathrm{OH}^{-}}{\rightarrow}\left[\mathrm{CoEn}_{2}(\mathrm{SCN})(\mathrm{OH})\right]^{+} \quad 75 \quad 25
\end{aligned}
$$

showing that substitution in octahedral complexes proceeds predominantly, but not exclusively, by 'edge-displacement'. This result should be contrasted with $S_{N} 2$ substitution among tetrahedral carbon compounds, which leads practically exclusively to optical inversion.

The next speaker, Prof. F. S. Dainton (Leeds), prefaced his paper entitled "Physico-chemical Mechanisms involved in Electron Transfer" by saying that he proposed to underline some of the general remarks made by Dr. Irving. Atom transfers are certainly known, such as

$$
\left(\mathrm{S}^{18} \mathrm{O}_{3}\right)^{\prime \prime}+\left(\mathrm{Cl}{ }^{18} \mathrm{O}_{3}\right)^{\prime} \rightarrow\left(\mathrm{S}^{16} \mathrm{O}_{2}{ }^{18} \mathrm{O}\right)^{\prime \prime}+\left(\mathrm{Cl}{ }^{18} \mathrm{O}_{2}{ }^{18} \mathrm{O}\right)^{\prime} ;
$$

and electron transfers between, for example, argon and sodium vapour are well established. But while Tlt and $\mathrm{Tl}^{3+}$, and $\mathrm{Fe}^{2+}$ and $\mathrm{Fe}^{3+}$ give couples individually reversible at a platinum electrode so that the position of ultimate equilibrium in a mixture can be calculated from the redox potentials, these give no clue to the rate of reaction.

The fundamental principles of elèctron transfer reactions were then discussed by Prof. Dainton along the lines suggested by Mulliken, and by Evans and his co-workers. Vinyl compounds which are polymerized by hydrogen atoms serve as detectors for hydrogen atoms produced, for example, by photochemical electron-transfer from reducing ions, though it is always necessary to take into account possible complex formation between cations present and the 
vinyl compound used; acrylonitrile displaces the potential of the $\mathrm{V}^{2+} / \mathrm{V}^{3+}$ couple by as much as $0.78 \mathrm{~V}$. due to preferential complexing with the vanadous form. Prof. Dainton reviewed the relationship between ionization potential and electron transfer spectra in considerable detail and discussed the effects of complexing the reducing ion. He concluded with an exposition of contemporary theoretical views on the process of electron transfer in systems of the type $A^{z A} \ldots X \ldots B^{z B}$ in which $X$ forms a conducting path, and paid particular attention to the possibilities of tunnelling effects and to changes in the entropy of hydration caused by valency changes in the cations $A$ and $B$.

Dr. J. H. Baxendale described current work at the University of Manchester in his paper on "Some Aqueous Oxidation-Reduction Reactions". The low probability of termolecular reactions suggests that a non-symmetrical redox reaction, such as

$$
\mathrm{Tl}^{+}+2 \mathrm{Fe}^{3+} \rightleftharpoons \mathrm{Tl}^{3+}+2 \mathrm{Fe}^{2+},
$$

which formally involves a two-electron transfer, might proceed through intermediates having abnormal valency, for example, $\mathrm{Tl}^{2+}$. Higginson et al. have shown that the oxidation of hydrazine takes different courses, depending on the oxidant employed. Using nitrogen-15, it has been confirmed that there are two alternative reaction paths :

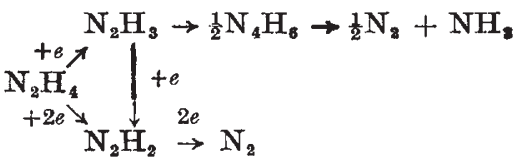

which may be taken, depending upon whether the oxidant used reacts in the first step by accepting a single electron, or two electrons simultaneously.

With hydrogen peroxide there is again a possibility of two reaction paths, depending upon conditions; for this substance may either be oxidized quantitatively to water and oxygen, or it may bo induced to undergo catalytic decomposition by way of self oxidation-reduction. Using reagents of high redox potential, a detailed study has been made of rates and mechanisms. In almost all cases the rate equation contains two terms, one of which involves the reciprocal of the hydrogen-ion concentration. The kinetic dependence on $p H$ in the case of oxidation by cobaltic ions is consistent with any one of the following mochanisms :

$$
\begin{aligned}
& \mathrm{Co}^{3+}+\mathrm{HO}_{2}^{-} \rightarrow \mathrm{Co}^{2+}+\mathrm{HO}_{2} \text { (electron transfer) } \\
& \mathrm{CoOH}^{2+}+\mathrm{H}_{2} \mathrm{O}_{2} \rightarrow \mathrm{Co}\left(\mathrm{H}_{2} \mathrm{O}\right)^{2+}+\underset{\text { (atom transfer) }}{\mathrm{HO}_{2}} \\
& \mathrm{CoO}_{2} \mathrm{H}^{2+} \rightarrow \mathrm{Co}^{2+}+\mathrm{HO}_{2} \text { (dissociation), }
\end{aligned}
$$

illustrating the great difficulties which attend the interpretation of kinetic data and the need for more detailed information concerning the stability and probable behaviour of metal complexes in solution.

The last part of Dr. Baxendale's paper dealt with the mechanism and kinetics of reactions of ferrousferric and thallous-thallic couples with some quinonehydroquinone systems. While the kinetics are consistent with reactions proceeding through a reversible one-electron step involving an ion of abnormal valency (for example, $\mathrm{Tl}^{2+}$ ), followed by a second one-electron step, there is once again the common ambiguity as to whether electron transfer, or atom transfer, or the transfer of a group is involved.
After a break, the symposium was resumed under the chairmanship of Dr. J. Chatt. The first paper, on the "Disproportionation Reactions of the Heavy Elements in Aqueous Solution", was given by Dr. R. Flurst (Harwell), who discussed the oxidationreduction potentials of couples formed by different valency states of uranium, neptunium, plutonium and americium. In every case the formal redox potentials are greatly influenced by the nature and concentration of acids present, pointing to extensive complex formation, while the closeness of values for different oxidation states points to their possible co-existence in solution. Thus plutonium exists in $0.05 M$ porchloric acid to the extent of $10,3,24$ and 63 per cent in the tri-, tetra-, penta- and hexa-valent states respectively, and disproportionation reactions, such as

$$
\begin{aligned}
\mathrm{PuO}_{2}^{+}+\mathrm{Pu}^{4+} & \rightleftharpoons \mathrm{PuO}_{2}^{++}+\mathrm{Pu}^{8+} \\
4 \mathrm{H}^{+}+\mathrm{PuO}_{2}^{+}+\mathrm{PuO}_{2}^{+} & \rightleftharpoons \mathrm{Pu}^{4+}+\mathrm{PuO}_{2}^{++}+2 \mathrm{H}_{2} \mathrm{O} \\
4 \mathrm{H}^{+}+\mathrm{PuO}_{2}^{+}+\mathrm{Pu}^{3+} & \rightleftharpoons 2 \mathrm{Pu}^{4+}+2 \mathrm{H}_{2} \mathrm{O},
\end{aligned}
$$

proceeding at different speeds, further complicate the picture. The interacting species are less simple even than already suggested, for from the effect of changing hydrogen-ion concentration on the rate of the backreaction in the third equation, it would appear that the two tetravalent plutonium ions ought rather to be differentiated as $\mathrm{Pu}(\mathrm{OH})^{3+}$ and $\mathrm{Pu}(\mathrm{OH})_{2}{ }^{2+}$, respectively. When studying the solution chemistry of the heavier radioactive elements, still other complications arise from side-reactions induced by the effects of intense radiation on the solvents and reactants.

Dr. K. W. Sykes (Swansea), who followed, further emphasized the importance of an exact knowledge of the concentration and nature of the species present before embarking on attempts to elucidate reaction kinetics. Although perchlorates have almost universally been used as standards of non-association, he questioned the validity of assuming that complexformation between cations and perchlorate ions is always negligible. Using spectrophotometric methods, he investigated the interaction of ferric ions with perchlorate, sulphate, bisulphate and hydroxyl ions, taking into account the possible formation of an equilibrium mixture of all the possible binary complexes, as well as mixed complexes such as $\mathrm{Fe}(\mathrm{OH})\left(\mathrm{ClO}_{4}\right)^{2-k}$. The value found for the stability constant $\left(\mathrm{FeClO}_{4}^{2+}\right) /\left(\mathrm{Fe}^{3+}\right)\left(\mathrm{ClO}_{4}^{-}\right)=19(\mu=0)$ in. dicates considerable association, a result consistent with most other spectrophotometric studies and with the effect of perchlorate ion on the kinetics of the ferric iodide reaction.

The last paper in the symposium, dealing with "Hydrolysis and Exchange Studies of some Chromic and Cobaltic Complexes", was presented by Dr. R. G. Wilkinson (Sheffield) and described work carried out in collaboration with Dr. A. W. Adamson (University of Southern California). For the aquation reaction

$$
\begin{aligned}
& {\left[\mathrm{Cr}\left(\mathrm{NH}_{3}\right)_{5}(\mathrm{SCN})\right]^{++}+\mathrm{H}_{2} \mathrm{O} \rightleftharpoons} \\
& {\left[\mathrm{Cr}\left(\mathrm{NH}_{3}\right)_{5}\left(\mathrm{H}_{2} \mathrm{O}\right)\right]^{+++}+\mathrm{SCN}^{-} \text {, }}
\end{aligned}
$$

the first-order rate constant was found to be $k=1.17 \times 10^{13} \exp (-24,900 / R T)$; but the rate of bimolecular exchange of thiocyanate ion with the thiocyanatopentammine containing the labelled group $\mathrm{S}^{14} \mathrm{CN}$ was found to be not more than a few per cent of the aquation-rate and undetectable against the 
aquation background. For the first-order aquation of the corresponding cobaltammine, however, $\left[\mathrm{Co}\left(\mathrm{NH}_{3}\right)_{5}(\mathrm{SCN})\right]^{++}+\mathrm{H}_{2} \mathrm{O} \rightleftharpoons$

$$
\left[\mathrm{Co}\left(\mathrm{NH}_{3}\right)_{5}\left(\mathrm{H}_{2} \mathrm{O}\right)\right]^{+++}+\mathrm{SCN}^{-}
$$

the rate was given by $k=0.85 \times 10^{13}$ exp $(-26,800 / R T)$. While in this case aquation and the rate of release of thiocyanate ions was found to be catalysed by thiocyanate ions, no similar rateenhancement was observed with the chromammine. These differences could be explained by postulating the operation of an association $\left(S_{N} 2\right)$ mechanism for the chromium complexes, but a dissociation $\left(S_{N} 1\right)$ mechanism for those of cobalt. But it should not be overlooked that the rate of exchange of water is known to be increased in many cases by increase in ionic strength, so that the catalytic effect of the thiocyanate ions might well be due to their causing an increase in ionic strength rather than to the progress of bimolecular substitution.

The lively and prolonged discussions which followed each group of papers brought valuable contributions from a very considerable number among the audience and added greatly to the success of what was obviously an exceptionally well-appreciated, stimulating, and well-timed symposium. Since the whole of these discussions is to be reported in full by the Chemical Society in a special publication of all the papers contributed to the symposium, no attempt will be made here to indicate even their scope or quality; but they certainly suggested that the hopes expressed by the president in his opening remarks would not long await realization.

\section{H. Irving}

\section{UGANDA PROTECTORATE FOREST DEPARTMENT}

\section{ANNUAL REPORT FOR 1952}

$\mathrm{T}$

HE annual report of the Uganda Forest Department for the year ending December $1952^{*}$ makes an outstanding departure from the forestry reports of most of the British Colonies-and, it might be added, other parts of the Commonwealth-by virtue of the noteworthy position it gives to a consideration of working plans. This important branch of forestry work is dealt with under the heading of "Enumerations and Working Plans" in Chapter 3, and follows remarks on policy and legislation in Chapter 1 , the form of which remains unchanged and on the lines of the official statement made by the Governor in 1948, and data on the forest estate for the year, which are contained in Chapter 2.

A working plan for a forest is not necessarily made only when an annual financial income is assured. A chancellor of the exchequer or financial minister to a Colonial Government has to provide money in his budget for non-productive objects of indirect benefit to the community. In a similar fashion, forests may have to be managed, especially protection forests or those which secure amenities for the public but do not make a direct financial profit. As regards financially productive forests, a working plan is necessary even though the area may be run on a short rotation for the public,

- Uganda. Annual Report of the Forest Department for the Year ended 31st December, 1952. Pp. il 38. (Entebbe: Government Printer, 1963.) 1.758. often to provide the agricultural villager with fuel. Once under a working plan sanctioned by the highest Civil authority in the country, a forest is safe in perpetuity so long as the prescriptions laid down for a short period of years are strictly carried out. A perusal of the chapter in this report devoted to this branch of forestry work shows that these premises are well understood by the Government and the Chief Conservator of Forests in Uganda. Equally interesting are the remarks on the methods upon which the enumerations of the growing stock of the forests are being undertaken; for a plan cannot be drawn up until as detailed a knowledge of the contents of the forest as possible is obtained.

Five new working plans were approved during the year, and progress was made in the compilation of a number of other plans and revisions, some being in first draft during the year. One of the sanctioned plans, the Mbale Plantations for 1953-60, is for pole and fuel plantations covering a coppice rotation ; another prescribes the management of six protection central forest reserves in the northern Acholi hills; a third, the West Nile Southern Escarpment hilltracts working plan for 1952-61, prescribes the protection and maintenance of nine local forest reserves in the West Nile. The mere names and the reasons for these working plans indicate the sound lines of work which the Chief Conservator is engaged upon.

It is all the more satisfactory and of promise for the future that working plans are in force or in preparation for local forests under African management. The area of pole or fuel plantations, twelve in number, managed by African local governments amount to 7,691 acres, of which several are already under working plans. This type of work in tropical forests involves a considerable amount of research, and the staff of the Department are to be congratulated on the amount of work which has been carried out on this account. E. P. STrebring

\section{CARNEGIE INSTITUTION OF WASHINGTON \\ ANNUAL REPORT}

$\mathrm{D}^{\mathrm{H}}$ R. VANNEVAR BUSH'S annual report* as president of the Carnegie Institution of Washington mentions as the most interesting of the Institution's research activities in the year ending June 30, 1953, an entirely new approach at the Mount Wilson and Palomar Observatories to the problem of the general magnetic field of the sun. Observations made possible by the greatly increased power of the new Mount Wilson gratings, and new photoelectric techniques have confirmed the existence of a general magnetic field much weaker than earlier measurements suggested and limited to the neighbourhood of the poles. Preliminary values obtained with the new Hale telescope and more advanced techniques now available indicate that $a_{\text {drastic }}$ revision of the distance of the Andromeda nebula and other distances dependent on it will be required : the earlier values of the distances and linear dimensions of all nebulæ must be approximately doubled

* California Institute of Technology. Annual Report 1952-1953. Comprising the Reports of the President, the Secretary, the Comptroller, and other Administrative Officers. Pp. v+142. (Pasadena, Calif. : California Institute of Technology. 1953.) 